\title{
The presence of emphysema on chest imaging and mid-life cognition
}

\author{
Benjamin E. Henkle $\mathbb{1}^{1,2}$, Laura A. Colangelo ${ }^{3}$, Mark T. Dransfield ${ }^{4}$, Lifang Hou ${ }^{3}$, \\ David R. JacobsJr $\mathbb{1}^{5}$, Brian T. Joyce ${ }^{3}$, Carrie L. Pistenmaa $\mathbb{1}^{6}$, Rachel K. Putman $\mathbb{1}^{6}$, \\ Steve Sidney ${ }^{7}$, Bharat Thyagarajan $\mathbb{1}^{8}$, George R. Washko ${ }^{6}$, Kristine Yaffe ${ }^{9}$, \\ Ravi Kalhan ${ }^{3,10}$ and Ken M. Kunisaki ${ }^{1,2,10}$
}

\begin{abstract}
Affiliations: ${ }^{1}$ Minneapolis Veterans Affairs Health Care System, Minneapolis, MN, USA. ${ }^{2}$ Division of Pulmonary, Allergy, Critical Care and Sleep, University of Minnesota, Minneapolis, MN, USA. ${ }^{3}$ Northwestern University Feinberg School of Medicine, Chicago, IL, USA. ${ }^{4}$ Division of Pulmonary, Allergy, and Critical Care Medicine, University of Alabama at Birmingham, Lung Health Center, Birmingham, AL, USA. ${ }^{5}$ Division of Epidemiology and Community Health, University of Minnesota, School of Public Health, Minneapolis, MN, USA. ${ }^{6}$ Division of Pulmonary and Critical Care Medicine, Brigham and Women's Hospital, Boston, MA, USA. ${ }^{7}$ Division of Research, Kaiser Permanente, Oakland, CA, USA. ${ }^{8}$ Dept of Laboratory Medicine and Pathology, University of Minnesota, Minneapolis, MN, USA. ${ }^{9}$ Depts of Psychiatry, Neurology and Epidemiology, University of California San Francisco, San Francisco, CA, USA. ${ }^{10}$ These authors contributed equally.
\end{abstract}

Correspondence: Benjamin E. Henkle, Minneapolis VA Health Care System, Pulmonary and Sleep Medicine (111N), 1 Veterans Drive, Minneapolis, MN55417, USA. E-mail: henkl004Qumn.edu

\section{ABSTRACT}

Background: Airflow obstruction is associated with cognitive dysfunction but studies have not assessed how emphysema, a structural phenotype of lung disease, might be associated with cognitive function independent from pulmonary function measured by spirometry. We aimed to determine the relationship between the presence of visually detectable emphysema on chest computed tomography (CT) imaging and cognitive function.

Methods: We examined 2491 participants, mean age of 50 years, from the Coronary Artery Risk Development in Young Adults study who were assessed for the presence of emphysema on chest CT imaging and had cognitive function measured 5 years later with a battery of six cognitive tests.

Results: Of those assessed, 172 (7\%) had emphysema. After adjusting for age, sex, height, study centre, race, body mass index, education and smoking, visual emphysema was significantly associated with worse performance on most cognitive tests. Compared to those without emphysema, participants with emphysema performed worse on cognitive testing: 0.39 SD units lower (95\% CI $-0.53--0.25)$ on the Montreal Cognitive Assessment, 0.27 SD units lower (95\% CI -0.42--0.12) on the Rey Auditory Verbal Learning Test, 0.29 SD units lower $(95 \%$ CI $-0.43--0.14)$ on the Digit Symbol Substitution Test and 0.25 SD units lower (95\% CI -0.42- -0.09) on letter fluency. Further adjustment for forced expiratory volume in $1 \mathrm{~s}\left(\mathrm{FEV}_{1}\right)$, peak $\mathrm{FEV}_{1}$ and annualised $\mathrm{FEV}_{1}$ decline did not attenuate these associations.

Conclusions: The presence of emphysema on chest CT is associated with worse cognitive function, independent of airflow obstruction. These data suggest that emphysema may be a novel risk factor for cognitive impairment.

@ERSpublications

Visual emphysema on thoracic CT imaging is associated with worse cognitive test performance. This association is independent of lung function and suggests that emphysema may be a novel risk factor for cognitive impairment. https://bit.ly/2LBTYaE

Cite this article as: Henkle BE, Colangelo LA, Dransfield MT, et al. The presence of emphysema on chest imaging and mid-life cognition. ERJ Open Res 2021; 7: 00048-2021 [https://doi.org/10.1183/ 23120541.00048-2021].
This article has supplementary material available from openres.ersjournals.com

Received: 22 Jan 2021 | Accepted: 4 Feb 2021

The content of this work is not subject to copyright. Design and branding are copyright OERS 2021. This version is distributed under the terms of the Creative Commons Attribution Non-Commercial Licence 4.0. For commercial reproduction rights and permissions contact permissions@ersnet.org 


\section{Introduction}

Dementia and cognitive impairment are growing public health priorities $[1,2]$. With no effective disease-modifying treatments for cognitive impairment, public health efforts are focused on prevention and identification of at-risk individuals with modifiable risk factors [3]. Impaired lung function has been identified as a potentially modifiable risk factor for future cognitive dysfunction [4].

Several studies have shown an association between cognitive impairment and measures of lung function like forced vital capacity (FVC), forced expiratory volume in $1 \mathrm{~s}\left(\mathrm{FEV}_{1}\right)$ and lung function decline [4-6]. In older adults, chronic obstructive pulmonary disease (COPD) has also been associated with cognitive dysfunction [7, 8]. Numerous mechanistic pathways have been proposed including hypoxaemia, systemic inflammation, vascular disease, smoking and physical inactivity [9].

In addition to traditional spirometry-based assessments of lung disease, emerging data suggest that computed tomography (CT) assessments provide valuable clinical information on structural lung changes, especially in regard to emphysema. CT assessments reveal structural lung changes that may be present even in the absence of clinical symptoms or spirometry abnormalities.

CT-based emphysema phenotypes have been associated with distinct differences in survival patterns, with worse mortality among those with greater extent of emphysema [10]. Individuals with confluent or advanced destructive emphysema have almost twice the mortality risk as those with trace or mild centrilobular emphysema [10]. Further, different types of chest CT emphysema measures have been associated with increased frequency of COPD exacerbations [11], worse quality of life [12] and more rapid lung function decline $[13,14]$.

To our knowledge, the association between cognitive function and the presence of emphysema on chest CT imaging has not been assessed. We addressed this knowledge gap by using data from the Coronary Artery Risk Development in Young Adults (CARDIA) study and hypothesised that those with an emphysema phenotype would have worse cognitive function.

\section{Methods}

A complete description of our methods is provided in the supplementary material.

\section{Study design, setting and participants}

CARDIA is a prospective cohort study that enrolled 5115 black and white participants aged 18-30 years old in 1985-1986 from communities in Birmingham, AL, Chicago, IL, Minneapolis, MN, and Oakland, CA, USA [15]. Follow up visits were conducted at years 2, 5, 7, 10, 15, 20, 25 and 30. The study was approved by institutional review boards at each centre. Written informed consent was obtained from all participants.

\section{Predictor: chest CT emphysema}

At study year 25, participants underwent chest CT scans. Visual assessment of emphysema was performed by two radiologists (with a third radiologist for adjudication when necessary) using methods previously described [16-19]. The $\kappa$ agreement between readers 1 and 2 for centrilobular emphysema was 0.74 , and for paraseptal emphysema was 0.70 .

\section{Outcomes: cognitive assessment}

At study year 30, participants completed six cognitive tests administered by trained interviewers: the Montreal Cognitive Assessment (MoCA), which is a screening test for mild cognitive impairment; the Rey Auditory Verbal Learning Test (RAVLT), which assesses verbal memory; the Digit Symbol Substitution Test (DSST), which assesses processing speed; the Stroop Test, which is a test of executive function; and tests of verbal fluency (category and letter fluency), which assess verbal ability and executive control. Raw scores from each test were transformed into standardised $\mathrm{z}$-scores, where positive $\mathrm{z}$-scores reflect better results. MoCA scores < 26 are clinically validated and suggest mild cognitive impairment [20]. Additional information regarding the cognitive assessments is available in the supplemental material.

\section{Other data}

Pre-bronchodilator spirometry was performed at study years $0,2,5,10,20$ and 30 in accordance with procedures recommended by the American Thoracic Society [21-24]. Usual physical activity was self-reported using the CARDIA Physical Activity Questionnaire [25].

\section{Statistical analysis}

Participants were excluded if they were missing chest CT or spirometry data, or if they were missing more than two cognitive function scores. 
Multivariable linear regression was used to determine the association between visual emphysema on chest CT imaging and cognitive dysfunction. Model covariates included age, sex, race, study centre, height, body mass index (BMI), years of education, smoking status (current, former and never), smoking pack years at year 25, and cigarettes per day among current and former smokers. Additional multivariable linear regression models adjusting for each $\mathrm{FEV}_{1}$, peak $\mathrm{FEV}_{1}$ and annualised $\mathrm{FEV}_{1}$ decline were developed to assess the association between visual emphysema and cognitive test performance independent of spirometry. To further explore effects on global cognitive function measured by the MoCA, a multivariable logistic regression model was used. We also conducted analyses stratified by smoking exposures.

A post hoc analysis to explore potential mediators of the association between emphysema and cognition was conducted using the "Mediation" package in R 4.0.0 (R Core Team, 2020). For models testing the association between emphysema and cognition, we analysed mediation by emphysema-induced systemic inflammation by inserting year 25 plasma C-reactive protein (CRP) as mediator. We also explored physical activity as a mediator using year 25 total activity scores derived from the CARDIA Physical Activity Questionnaire.

Analyses were conducted using SAS 14.1 (SAS Institute, Cary, NC, USA) and R 4.0.0.

\section{Results}

\section{Participant characteristics}

Among 5115 CARDIA participants at baseline, we included 2491 study participants in this analysis. Reasons for exclusion are summarised in figure 1 but the main reason for exclusion was failure to attend the year 25 or year 30 study visit. A total of $172(7 \%)$ participants had visual emphysema on chest CT imaging. Of those with emphysema, 106 (62\%) had centrilobular emphysema, 131 (76\%) had paraseptal emphysema and $65(38 \%)$ had both. Compared to participants without emphysema, those with emphysema were more likely to be of black race and male sex, had fewer years of education, and increased smoking burden (table 1). FVC was similar between those with and without emphysema but $\mathrm{FEV}_{1}$ and $\mathrm{FEV}_{1} \%$ predicted were significantly lower among those with emphysema (table 1).

\section{Emphysema and cognition}

In unadjusted analyses, there were statistically significant associations between the presence of emphysema and cognitive testing by the MoCA, RAVLT, DSST, Letter Fluency, Stroop and Category Fluency tests (table 2 and e-table 1). After adjusting for potential confounders of this relationship (age, sex, height, study centre, race, BMI, education, current cigarettes per day and pack-years of smoking), visual emphysema continued to be significantly associated with 0.39 SD units lower performance on the MoCA (95\% CI $-0.53--0.25), 0.27$ SD units lower performance on the RAVLT (95\% CI $-0.42--0.12$ ), 0.29 SD units lower performance on the DSST (95\% CI $-0.43--0.14$ ) and 0.25 SD units lower performance on letter fluency (95\% CI $-0.42--0.09$ ) (figure 2 and e-table 1).

Further adjustment for $\mathrm{FEV}_{1}$, peak $\mathrm{FEV}_{1}$ or annualised $\mathrm{FEV}_{1}$ decline did not attenuate these associations (figure 2 and e-table 1).

Of the 172 participants with emphysema, 148 (86\%) had a MoCA score <26 (the clinical screening threshold for possible mild cognitive impairment) compared to 1336 (57.6\%) out of 2319 without emphysema. After adjusting for confounders, having emphysema was associated with increased odds of

FIGURE 1 Numbers of participants included in the analyses presented based on availability of chest computed tomography (CT) imaging, cognitive function testing and spirometry. CRP: C-reactive protein.
Exclusion:

1 drop out

1756 did not attend year 30 visit

298 did not attend year 25 visit

255 missing CT emphysema scoring

131 missing spirometry

109 missing $>2$ cognitive assessments

51 missing year 25 covariates

23 missing mediator (CRP or total activity score)

5115 participants

2491 participants included in analysis 
TABLE 1 Demographic and clinical characteristics at the year 25 study visit and spirometry at the year 30 study visit

\begin{tabular}{|c|c|c|}
\hline & Emphysema & No emphysema \\
\hline Subjects & 172 & 2319 \\
\hline Age years & $50 \pm 3.5$ & $50 \pm 3.6$ \\
\hline Female & $77(45)$ & $1350(58)$ \\
\hline \multicolumn{3}{|l|}{ Race } \\
\hline Black & 115 (67) & $1031(44)$ \\
\hline White & 57 (33) & $1288(56)$ \\
\hline \multicolumn{3}{|l|}{ Clinical centre } \\
\hline Birmingham & $38(22)$ & $550(24)$ \\
\hline Chicago & 32 (19) & $518(22)$ \\
\hline Minneapolis & 57 (33) & $569(25)$ \\
\hline Oakland & $45(26)$ & $682(29)$ \\
\hline Height $\mathrm{cm}$ & $171 \pm 9.1$ & $170 \pm 9.4$ \\
\hline BMI $\mathrm{kg} \cdot \mathrm{m}^{-2}$ & $27 \pm 5.5$ & $30 \pm 7.1$ \\
\hline Education years & $13 \pm 2.4$ & $15 \pm 2.6$ \\
\hline Pack years at year 25 & $20 \pm 15$ & $4.0 \pm 8.4$ \\
\hline Cigarettes per day among current and former smokers only & $8.2 \pm 9.3$ & $1.2 \pm 4.1$ \\
\hline \multicolumn{3}{|l|}{ Smoking status } \\
\hline Never & $26(15)$ & $1542(66)$ \\
\hline Former & $36(21)$ & $515(22)$ \\
\hline Current & $110(64)$ & $262(11)$ \\
\hline \multicolumn{3}{|l|}{ Spirometry } \\
\hline $\mathrm{FEV}_{1} \mathrm{~L}$ & $2.45 \pm 0.79$ & $2.74 \pm 0.75$ \\
\hline FEV $1 \%$ predicted & $82.6 \pm 20.3$ & $92.8 \pm 15.5$ \\
\hline FVC L & $3.5 \pm 0.94$ & $3.6 \pm 0.98$ \\
\hline FVC $\%$ predicted & $92.3 \pm 15.9$ & $94.0 \pm 14.5$ \\
\hline $\mathrm{FEV}_{1} / \mathrm{FVC}$ ratio & $0.70 \pm 0.11$ & $0.77 \pm 0.06$ \\
\hline
\end{tabular}

Data are presented as mean \pm SD or $n(\%)$, unless otherwise stated. BMI: body mass index; $\mathrm{FEV}_{1}$ : forced expiratory volume in $1 \mathrm{~s}$; FVC: forced vital capacity.

having a MoCA score <26 (OR 2.47, 95\% CI 1.48-4.11). Further adjustment for $\mathrm{FEV}_{1}(\mathrm{OR} 2.35,95 \% \mathrm{CI}$ 1.41-3.92), peak $\mathrm{FEV}_{1}$ (OR 2.45, 95\% CI 1.47-4.07) or annualised $\mathrm{FEV}_{1}$ decline (OR 2.45, 95\% CI 1.45-4.16) only modestly decreased the odds of having a MoCA score <26 (e-table 2).

\section{TABLE 2 Cognitive assessments from examination year 30}

\begin{tabular}{|c|c|c|}
\hline Cognition & Emphysema" & No emphysema" \\
\hline \multicolumn{3}{|l|}{ Raw score } \\
\hline $\mathrm{MoCA}$ & $21.0 \pm 4.2$ & $24.2 \pm 3.7$ \\
\hline RAVLT & $6.4 \pm 3.4$ & $8.7 \pm 3.3$ \\
\hline DSST & $56.1 \pm 16.9$ & $69.1 \pm 16.0$ \\
\hline Letter fluency & $35.7 \pm 12.5$ & $42.4 \pm 12.8$ \\
\hline Stroop $^{+}$ & $27.8 \pm 12.4$ & $22.4 \pm 11.1$ \\
\hline Category fluency & $18.5 \pm 5.4$ & $20.5 \pm 5.4$ \\
\hline \multicolumn{3}{|l|}{ z-score } \\
\hline MoCA & $-0.78 \pm 1.1$ & $0.06 \pm 0.97$ \\
\hline RAVLT & $-0.63 \pm 1.0$ & $0.05 \pm 0.98$ \\
\hline DSST & $-0.74 \pm 1.0$ & $0.06 \pm 0.98$ \\
\hline Letter fluency & $-0.49 \pm 0.97$ & $0.03 \pm 0.99$ \\
\hline Stroop ${ }^{+, \S}$ & $-0.45 \pm 1.1$ & $0.03 \pm 0.98$ \\
\hline Category fluency & $-0.35 \pm 0.99$ & $0.03 \pm 1.00$ \\
\hline \multicolumn{3}{|c|}{$\begin{array}{l}\text { Data are presented as mean } \pm \text { SD. MoCA: Montreal Cognitive Assessment; RAVLT: Rey Auditory Verbal } \\
\text { Learning Test; DSST: Digit Symbol Substitution Test. }{ }^{\#}: n=172 .{ }^{\uparrow}: n=2319 ;^{+}: \text {Stroop interference scores are } \\
\text { reported; in contrast to other cognitive tests, higher Stroop scores reflect worse cognitive performance; } \\
\S \text { : Stroop interference score has been reported as an inverse score for consistency with other tests. }\end{array}$} \\
\hline
\end{tabular}


Among those with centrilobular emphysema, there were statistically significant differences between emphysema and the MoCA, RAVLT and letter fluency tests, but not the DSST, Stroop or category fluency tests (e-table 3). In those with paraseptal emphysema, statistically significant differences were seen in the MoCA and DSST tests (e-table 3).

In analyses stratified by smoking status, there was no association between emphysema and cognitive function amongst current smokers, but relationships remained significant in former and never-smokers (e-table 4). When stratified by pack-years of smoking, associations were similar in those with 0 pack-years (never-smokers) but inconsistent in those with $>0-10$ and $>10$ pack-years of smoking (e-table 5).

\section{Mediator analysis}

To explore whether the association between emphysema and cognitive dysfunction could be mediated through systemic inflammation or physical activity, we obtained estimates of the direct and indirect effects of emphysema on cognitive function measures. There were statistically significant indirect effects of emphysema on cognitive performance through CRP on cognition measured by DSST. The estimate of the indirect effect was -0.21 (95\% CI -0.44- -0.04) with per cent mediated $4.3 \%$ (95\% CI $0.7-12.0 \%)$. CRP did not act as a mediator for any other cognitive assessments including the MoCA, RAVLT, letter fluency, Stroop or category fluency tests (e-table 6). There were no indirect effects of emphysema on cognitive function measured by the MoCA, RAVLT, DSST, letter fluency, Stroop or category fluency tests through the total physical activity score (e-table 7).

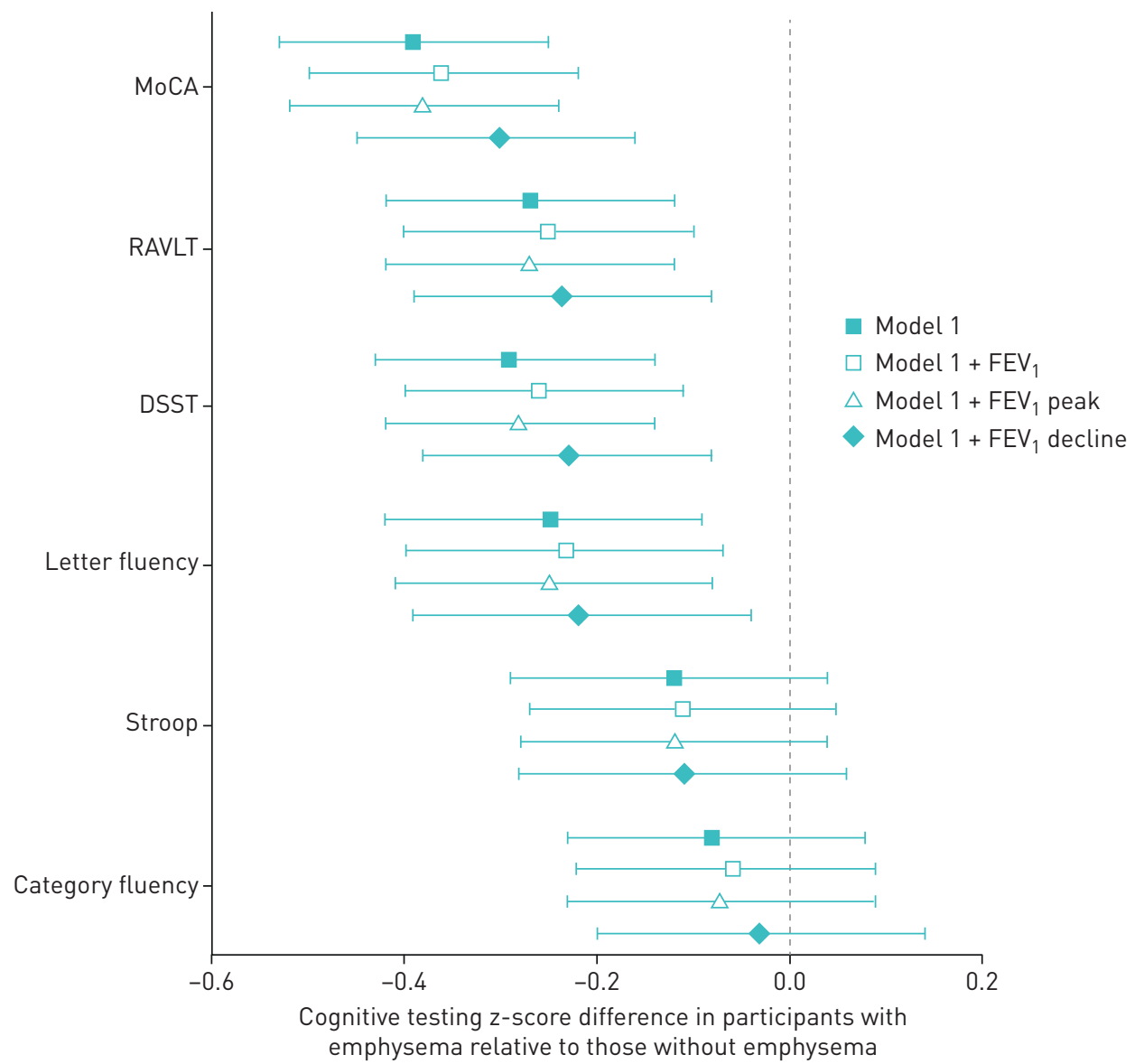

FIGURE 2 Differences in cognitive test z-scores between Coronary Artery Risk Development in Young Adults cohort participants with and without chest computed tomography visual emphysema. Shown for each cognitive test is the multivariable linear regression $\beta$ coefficient $195 \% \mathrm{Cl})$ for the association between emphysema and cognitive test $z$-score. Negative values reflect worse cognitive test performance, where -1.0 is a 1 standard deviation worse test score and +1.0 is a 1 standard deviation better test score. Model 1 covariates: year 25 age, sex, race, body mass index, height, study centre, education, cigarettes per day and pack years. Stroop interference scores are reported as an inverse score for consistency with other tests. In contrast to other cognitive tests, higher Stroop scores reflect worse cognitive performance. MoCA: Montreal Cognitive Assessment; RAVLT: Rey Auditory Verbal Learning Test; DSST: Digit Symbol Substitution Test; FEV ${ }_{1}$ : forced expiratory volume in $1 \mathrm{~s}$. 


\section{Discussion}

To our knowledge, the association between structural lung changes of emphysema visually detected on CT imaging and cognitive outcomes has not previously been assessed. Among middle-aged adults in this population-based cohort, we found that emphysema was associated with worse cognitive performance scores across several domains of cognition. The effect sizes of these associations were approximately 0.2-0.3 standard deviations worse for those with emphysema and remained fairly consistent across multiple assessments of cognitive function. These findings were detected despite the cohort being only 50 years old on average at the time of emphysema assessment, with few comorbidities and mild lung disease by traditional measures (mean $\mathrm{FEV}_{1} 83 \%$ predicted in those with emphysema). While the effect size is small to moderate, this may suggest that emphysema, even in middle-aged adults with normal lung function, is linked to cognitive performance.

We found a significant association between emphysema and impaired global cognitive function as measured by the MoCA. In the unadjusted analysis, individuals with emphysema scored on average 3 points $(\sim 0.8 \mathrm{SD})$ lower on the 30-point scale of the MoCA than those without emphysema. Furthermore, based on the standard clinical threshold of a MoCA score $<26$, significantly more of those with emphysema screened positive for mild cognitive impairment compared to those without emphysema.

While this study assessed only the presence of emphysema and not a diagnosis of COPD, mild cognitive impairment is common in the COPD population, and has implications in both disease management and patient quality of life. COPD patients with reduced fluid cognitive ability have lower rates of medication adherence and worse inhaler technique [26]. Others have shown that COPD and mild cognitive impairment have additive effects on the risk of disability in performing activities of daily living, and that the presence of these together increases risk more than each individually [27].

Several studies have shown a correlation between lung function measured by spirometry and cognition [9]. A meta-analysis of pulmonary function and dementia found a dose-response association between lung function and risk of dementia-related death with a hazard ratio (HR) of 2.09 (95\% CI 1.17-3.71) between the first and last quartiles of $\mathrm{FEV}_{1}$ after adjusting for age, sex, socioeconomic status, health behaviours including smoking, and illness burden [28]. In the Atherosclerosis Risk in Communities cohort, after controlling for age, sex, centre, education, race-centre and smoking, participants in the lowest quintile of $\mathrm{FEV}_{1} \%$ predicted and FVC \% predicted had an elevated risk of dementia compared to the highest quintile (HR 1.23 (95\% CI 1.04-1.47) and HR 1.36 (95\% CI 1.14-1.61)) [4]. Spirometric patterns of restriction and airflow obstruction have also been associated with dementia and mild cognitive impairment (OR 1.56 (95\% CI 1.12-2.16) and OR 1.31 (95\% CI 1.06-1.62) respectively) [4]. Others have shown that among individuals diagnosed with COPD, the degree of cognitive impairment is correlated with disease severity as categorised by $\mathrm{FEV}_{1}$ [29].

Importantly, we found that adjustment for $\mathrm{FEV}_{1}$, peak $\mathrm{FEV}_{1}$ and annualised $\mathrm{FEV}_{1}$ decline did not attenuate the association between emphysema and cognitive performance suggesting that emphysema may be a novel risk factor in the development of cognitive dysfunction in COPD.

Two major subtypes of emphysema are centrilobular emphysema and paraseptal emphysema, but little is known about the differing underlying pathophysiology leading to these distinct patterns of emphysema. Emerging evidence suggests these subtypes vary not only in clinical symptoms but also in pathological changes to the terminal bronchioles and leukocyte infiltration [30, 31]. In our analyses, centrilobular emphysema seemed to have a more durable association with cognitive performance than paraseptal emphysema, maintaining statistically significant associations with half of the cognitive assessments evaluated. These exploratory data suggest the need for further studies to understand how emphysema phenotypes and their individual underlying pathological mechanisms may contribute to changes in cognitive performance.

Smoking is one explanation for the relationship between emphysema and cognitive function. It has an established relationship with emphysema and has previously been shown to be associated with worse cognitive function $[32,33]$. In addition, others have shown that emphysema is found across a spectrum of individuals and may present in those with little or no smoking history [34]. To evaluate this further, we performed exploratory post hoc analyses, stratifying by smoking status and pack-years. While statistical power was reduced in these stratified analyses, they were notable for the strongest and most consistent associations being seen in never-smokers with emphysema rather than active or former heavy smokers with emphysema. This may be due to the effects of smoking (e.g. oxidative, pro-inflammatory and vascular damage) dominating over other emphysema-specific effects. The pathogenesis and clinical impact of nonsmoking emphysema is poorly understood but our exploratory analyses suggest potential clinical relevance of this entity and the need for improved understanding of emphysema pathogenesis in nonsmokers. 
Our observational study design did not allow us to address mechanisms but one possible pathway by which emphysema could lead to cognitive dysfunction is chronic systemic inflammation. The exact mechanisms underlying the development of emphysema are not well understood but it is accepted that COPD is associated with chronic inflammation of the airways and lung parenchyma [35]. CRP is one measure of systemic inflammation that has been associated with emphysema severity, progression and mortality [36]. Given these known relationships, we evaluated mediation effects of systemic inflammation as measured by plasma CRP on the association between cognitive function and emphysema. While there was evidence of mediation through CRP with cognition measured by DSST, no other tests displayed significant mediator effects. We acknowledge that this mediation analysis was only exploratory and CRP is a crude measure of systemic inflammation. Further studies using more sophisticated multi-omic pathway analysis may be required to better evaluate the potential role of systemic inflammation as a pathway by which emphysema might lead to cognitive impairment.

Another possible mediator of the association between emphysema and cognitive function is physical fitness. Physical function is associated with increased cognitive reserve and improved cognitive ageing, and may be a modifiable risk factor to reduce the risk of future cognitive impairment $[37,38]$. In addition, greater physical fitness in young adulthood is associated with less age-related lung function decline [39]. In our analysis, we did not find any mediation effects through total physical activity, but activity measures were self-reported, so these findings should be interpreted with caution.

Many other mechanisms might also explain how emphysema increases risk for cognitive decline. Future research should investigate potential mechanistic pathways such as vascular injury, cerebral small vessel disease, endothelial dysfunction, apoptosis, intermittent hypoxaemia and other more specific inflammatory pathways by which emphysema might contribute to cognitive deficits.

This study has several important limitations. Our design did not allow for longitudinal assessment of cognitive change, so we are unable to determine if those with emphysema experience a "one hit" defect in cognitive function that remains static over time or an "accelerated decline" process by which their cognitive function may continue to decline faster than those without emphysema. Such knowledge is important to determine the potential long-term clinical impact of emphysema on cognitive function. In addition, chest CT scans were acquired with cardiac gating, which prevented quantitative measures of emphysema [40] and we did not assess emphysema distribution or for the presence of coexisting interstitial lung abnormalities. Further characterisation and quantification of emphysema and other associated CT findings may help better determine how cognition and emphysema are related. Additionally, we were unable to assess how diffusion capacity is related to this association. Given the known relationship between emphysema and diffusion capacity, this should be considered in future studies. It is possible that CT measurement of emphysema may be impacted by cognition due to the need to image the lungs after receiving instructions to perform a maximal inhalation effort and then hold one's breath during image acquisition. However, reduced cognitive function would be associated with a poor breath-hold, which makes it more difficult to identify emphysema on CT and would likely bias our results toward the null. Lastly, our epidemiological study was not designed to address mechanisms. We performed mediation analyses but these results do not exclude the possibility that these factors are a link between cognition and emphysema. Further investigations are required to better understand causal pathways that could explain this relationship and to identify therapeutic targets that might reduce the risk of cognitive dysfunction in those with emphysema.

Our study's strengths include the large sample size of middle-aged adults, enrolment of participants from geographically diverse locations, good representation of women and African Americans, valid and reproducible spirometry measures, and the use of a battery of different well-established cognitive assessments that evaluate both global cognitive impairment and several cognitive domains.

\section{Conclusions}

In this large, population-based cohort of middle-aged adults, the presence of emphysema on chest CT at midlife was consistently associated with several domains of cognitive function, independent of lung function. This suggests that emphysema maybe a novel risk factor for cognitive impairment.

Acknowledgements: The authors thank the CARDIA participants.

Author contributions: B.E. Henkle, R. Kalhan and K.M. Kunisaki conceived and designed the study. R. Kalhan, S. Sidney and K. Yaffe obtained funding. L.A. Colangelo performed the primary statistical analyses. B.E. Henkle, R. Kalhan and K.M. Kunisaki drafted the manuscript. All authors critically revised the manuscript for important intellectual content and approved the final manuscript, and take responsibility for the integrity of the data and the accuracy of the data analysis. 
Conflict of interest: B.E. Henkle has nothing to disclose. L.A. Colangelo reports personal fees from ndd Medical Technologies outside the submitted work. M.T. Dransfield reports grants from the NIH, DoD, American Lung Association and Dept of Veteran Affairs; personal fees from AstraZeneca, Boerhringer Ingelheim, GlaxoSmithKline, Mereo, Pulmonx and PneumRx/BTG; and clinical trial support from AstraZeneca, Boehringer Ingelheim, Gala, GlaxoSmithKline, Nuvaira, Pulmonx, PneumRx/BTG and Yungjin, all outside the submitted work. L. Hou has nothing to disclose. D.R. Jacobs has nothing to disclose. B.T. Joyce has nothing to disclose. C.L. Pistenmaa reports grants from the Alpha 1 Foundation and Boehringer Ingelheim, and personal fees from Lancet Respiratory Medicine and Horizon, outside the submitted work. R.K. Putman reports grants from the National Institutes of Health outside the submitted work. S. Sidney has nothing to disclose. B. Thyagarajan has nothing to disclose. G.R. Washko reports serving on an advisory committee for Boehringer Ingelheim, CSL Behring, GlaxoSmithKline and Vertex; personal fees from Boehringer Ingelheim, CSL Behring, Janssen, Novartis, PulmonX and Vertex; serving on a data safety and monitoring board for PulmonX; received research support from Boehringer Ingelheim, BTG and Janssen, all outside the submitted work; has ownership and investment interest in Quantitative Image Solutions; and his spouse is an employee of Biogen K. Yaffe has nothing to disclose. R. Kalhan reports grants and personal fees from AstraZeneca, personal fees from CVS Caremark and Aptus Health, grants and personal fees from GlaxoSmithKline, and personal fees from Boston Scientific and the Boston Consulting Group, outside the submitted work. K.M. Kunisaki reports personal fees from GlaxoSmithKline and Nuvaira, Inc., and contracted clinical trial support from Sanofi, outside the submitted work.

Support statement: All authors have received grant support from the National Institutes of Health (NIH) for the work presented here. The Coronary Artery Risk Development in Young Adults Study (CARDIA) is conducted and supported by the National Heart, Lung, and Blood Institute (NHLBI) in collaboration with the University of Alabama at Birmingham (HHSN268201800005I and HHSN268201800007I), Northwestern University (HHSN268201800003I), the University of Minnesota (HHSN268201800006I) and the Kaiser Foundation Research Institute (HHSN268201800004I). Additional support was provided by R01 HL122477 (CARDIA Lung Study), R01 HL122658 and R01 AG063887 (CARDIA Cognition Study). CARDIA is also partially supported by the Intramural Research Program of the National Institute on Aging (NIA), and an intra-agency agreement between NIA and NHLBI (AG0005). This manuscript has been reviewed by CARDIA for scientific content. This material is also the result of work supported with resources and the use of facilities at the Minneapolis Veterans Affairs Medical Center, Minneapolis, USA. The views expressed in this article are those of the authors, and do not reflect the views of the US Government, the NIH, the Dept of Veterans Affairs, the funders, the sponsors or any of the authors' affiliated academic institutions. Funding information for this article has been deposited with the Crossref Funder Registry.

\section{References}

1 Alzheimer's Association, Centers for Disease Control and Prevention. The Healthy Brain Initiative: the Public Health Road Map for State and National Partnerships, 2013-2018. Chicago, Alzheimer's Association, 2013.

2 Matthews KA, Xu W, Gaglioti AH, et al. Racial and ethnic estimates of Alzheimer's disease and related dementias in the United States (2015-2060) in adults aged $\geqslant 65$ years. Alzheimers Dement 2019; 15: 17-24.

3 Nichols E, Szoeke CEI, Vollset SE, et al. Global, regional, and national burden of Alzheimer's disease and other dementias, 1990-2016: a systematic analysis for the Global Burden of Disease Study 2016. Lancet Neurol 2019; 18: 88-106.

4 Lutsey PL, Chen N, Mirabelli MC, et al. Impaired lung function, lung disease, and risk of incident dementia. Am J Respir Crit Care Med 2019; 199: 1385-1396.

5 Chyou PH, White LR, Yano $\mathrm{K}$, et al. Pulmonary function measures as predictors and correlates of cognitive functioning in later life. Am J Epidemiol 1996; 143: 750-756.

6 Anstey KJ, Windsor TD, Jorm AF, et al. Association of pulmonary function with cognitive performance in early, middle and late adulthood. Gerontology 2004; 50: 230-234.

7 Hung WW, Wisnivesky JP, Siu AL, et al. Cognitive decline among patients with chronic obstructive pulmonary disease. Am J Respir Crit Care Med 2009; 180: 134-137.

8 Singh B, Mielke MM, Parsaik AK, et al. A prospective study of chronic obstructive pulmonary disease and the risk for mild cognitive impairment. JAMA Neurol 2014; 71: 581-588.

9 Dodd JW, Getov SV, Jones PW. Cognitive function in COPD. Eur Respir J 2010; 35: 913-922.

10 Lynch DA, Moore CM, Wilson C, et al. CT-based visual classification of emphysema: association with mortality in the COPDGene study. Radiology 2018; 288: 859-866.

11 Han MK, Kazerooni EA, Lynch DA, et al. Chronic obstructive pulmonary disease exacerbations in the COPDGene study: associated radiologic phenotypes. Radiology 2011;261: 274-282.

12 Martinez $\mathrm{CH}$, Chen YH, Westgate PM, et al. Relationship between quantitative CT metrics and health status and BODE in chronic obstructive pulmonary disease. Thorax 2012; 67: 399-406.

13 Vestbo J, Edwards LD, Scanlon PD, et al. Changes in forced expiratory volume in 1 second over time in COPD. N Engl J Med 2011; 365: 1184-1192.

14 Galbán C, Han M, Boes J, et al. Computed tomography-based biomarker provides unique signature for diagnosis of COPD phenotypes and disease progression. Nat Med 2012; 18: 1711-1715.

15 Friedman GD, Cutter GR, Donahue RP, et al. CARDIA: study design, recruitment, and some characteristics of the examined subjects. J Clin Epidemiol 1988; 41: 1105-1116.

16 Hunninghake GM, Hatabu H, Okajima Y, et al. MUC5B promoter polymorphism and interstitial lung abnormalities. N Engl J Med 2013; 368: 2192-2200.

17 Washko GR, Lynch DA, Matsuoka S, et al. Identification of early interstitial lung disease in smokers from the COPDGene Study. Acad Radiol 2010; 17: 48-53.

18 Washko GR, Hunninghake GM, Fernandez IE, et al. Lung volumes and emphysema in smokers with interstitial lung abnormalities. N Engl J Med 2011; 364: 897-906.

19 Kalhan R, Dransfield MT, Colangelo LA, et al. Respiratory symptoms in young adults and future lung disease. The CARDIA Lung Study. Am J Respir Crit Care Med 2018; 197: 1616-1624.

20 Nasreddine ZS, Phillips NA, Bédirian V, et al. The Montreal Cognitive Assessment, MoCA: a brief screening tool for mild cognitive impairment. J Am Geriatr Soc 2005; 53: 695-699. 
ATS statement - Snowbird workshop on standardization of spirometry. Am Rev Respir Dis 1979; 119: 831-838. Standardization of spirometry - 1987 update. Statement of the American Thoracic Society. Am Rev Respir Dis 1987; 136: 1285-1298.

Lung function testing: selection of reference values and interpretative strategies. American Thoracic Society. Am Rev Respir Dis 1991; 144: 1202-1218.

Standardization of Spirometry, 1994 Update. American Thoracic Society. Am J Respir Crit Care Med 1995; 152: $1107-1136$.

Jacobs DR, Jr, Hahn LP, Haskell WL, et al. Validity and reliability of short physical activity history: cardia and the Minnesota Heart Health Program. J Cardiopulm Rehabil 1989; 9: 448-459.

O'Conor R, Muellers K, Arvanitis M, et al. Effects of health literacy and cognitive abilities on COPD self-management behaviors: a prospective cohort study. Respir Med 2019; 160: 105630.

Martinez CH, Richardson CR, Han MK, et al. Chronic obstructive pulmonary disease, cognitive impairment, and development of disability: the health and retirement study. Ann Am Thorac Soc 2014; 11: 1362-1370.

Russ TC, Starr JM, Stamatakis E, et al. Pulmonary function as a risk factor for dementia death: an individual participant meta-analysis of six UK general population cohort studies. J Epidemiol Community Health 2015; 69: $550-556$.

Crişan AF, Oancea C, Timar B, et al. Cognitive impairment in chronic obstructive pulmonary disease. PLoS One 2014; 9: e102468.

Tanabe N, Vasilescu DM, Hague CJ, et al. Pathological comparisons of paraseptal and centrilobular emphysema in chronic obstructive pulmonary disease. Am J Respir Crit Care Med 2020; 202: 803-811.

Smith BM, Austin JH, Newell JD, et al. Pulmonary emphysema subtypes on computed tomography: the MESA COPD study. Am J Med 2014; 127: 94.

Campos MW, Serebrisky D, Castaldelli-Maia JM. Smoking and cognition. Curr Drug Abuse Rev 2016; 9: 76-79.

Beydoun MA, Beydoun HA, Gamaldo AA, et al. Epidemiologic studies of modifiable factors associated with cognition and dementia: systematic review and meta-analysis. BMC Public Health 2014; 14: 643.

Hoffman EA, Ahmed FS, Baumhauer H, et al.. Variation in the percent of emphysema-like lung in a healthy, nonsmoking multiethnic sample. The MESA Lung Study. Ann Am Thorac Soc 2014; 11: 898-907.

5 Barnes PJ. Inflammatory mechanisms in patients with chronic obstructive pulmonary disease. J Allergy Clin Immunol 2016; 138: 16-27.

6 Zemans RL, Jacobson S, Keene J, et al. Multiple biomarkers predict disease severity, progression and mortality in COPD. Respir Res 2017; 18: 117.

Clouston SA, Brewster P, Kuh D, et al. The dynamic relationship between physical function and cognition in longitudinal aging cohorts. Epidemiol Rev 2013; 35: 33-50.

8 Zhu N, Jacobs DR, Jr, Schreiner PJ, et al. Cardiorespiratory fitness and cognitive function in middle age: the CARDIA study. Neurology 2014; 82: 1339-1346.

9 Benck LR, Cuttica MJ, Colangelo LA, et al. Association between cardiorespiratory fitness and lung health from young adulthood to middle age. Am J Respir Crit Care Med 2017; 195: 1236-1243.

Wells JM, Colangelo LA, Sivarajan L, et al. Inflammation and endothelial activation in early adulthood are associated with future emphysema: the CARDIA Lung Study. Eur Respir J 2019; 53: 1801532 